\title{
NUEVA ESPECIE GIPSÍCOLA DE PINGUICULA (LENTIBULARIACEAE) DEL ESTADO DE OAXACA, MÉXICO*
}

\author{
Sergio Zamudio Ruiz \\ Instituto de Ecología, A.C. \\ Centro Regional del Bajío \\ Apartado postal 386 \\ 61600 Pátzcuaro, Michoacán \\ Y \\ MilosLav StUdNiCKA \\ Botanická zahrada Liberec \\ Purkynova 1, 46001 Liberec 1 \\ Czech Republic
}

\section{RESUMEN}

Se describe como nueva especie a Pinguicula medusina que habita en colinas yesosas cercanas a Santiago Juxtlahuaca, Oaxaca. Por la corola subisoloba, con tubo cilíndrico y espolón corto se ubica en el subgénero Isoloba, sección Heterophyllum. Las "hojas de verano" largas y lineares la relacionan estrechamente con $P$. heterophylla Benth., de la que se separa por la forma y posición de los lóbulos de la corola, por la producción de yemas vegetativas en el ápice de las "hojas de verano" y por su adaptación para crecer en suelos yesosos.

\section{ABSTRACT}

Pinguicula medusina, inhabitant of gypsum hills near Santiago Juxtlahuaca, Oaxaca, is described as a new species. Because of its subisolobe corolla with cylindrical tube and short spur, it is placed in the subgenus Isoloba, section Heterophyllum. This new taxon is closely related to P. heterophylla Benth. in its long linear "summer leaves", but differs in the form and position of the corolla lobes, in the production of vegetative buds in the leaf apex and in its adaptation to grow on gypsum soils.

Uno de los problemas pendientes de resolver en el estudio del género Pinguicula en México es la diferenciación entre Pinguicula heterophylla Benth. y las plantas procedentes del estado de Oaxaca, conocidas por los horticultores con el nombre provisional de

\footnotetext{
* Trabajo realizado con apoyo económico del Instituto de Ecología, A.C. (cuenta 902-03), del Consejo Nacional de Ciencia y Tecnología, así como de la Comisión Nacional para el Conocimiento y Uso de la Biodiversidad.
} 
Pinguicula 'alfredae', así como la definición de la posición taxonómica correcta de estas últimas.

Al parecer, la nueva especie fue descubierta por A. Lau en 1977, cuando en compañía de J. Ríja y R. Subík exploraba unas colinas yesosas cercanas a Santiago Juxtlahuaca en busca de cactus; Lau colectó algunos individuos de esta Pinguicula y los envío a varios jardines botánicos de Europa. Uno de los primeros intentos por cultivar las plantas fue infructuoso, ya que las rosetas enviadas al Jardín Botánico de la University of Charles en Praga no se desarrollaron bien y se pudrieron en poco tiempo; sin embargo, en Linz, Austria se tuvo éxito en su cultivo y propagación y a partir de aquí se distribuyeron a otros lugares con el nombre superfluo de $P$. 'alfredae', sin definir su situación taxonómica.

Van Marm y Lampard (1992) consideraron que el dilema consistía en aclarar si $P$. 'alfredae' es sólo una forma de $P$. heterophylla o no; sin embargo, reconocieron que en ese momento no se contaba con suficiente material, ya sea vivo o preservado, como para tomar una decisión y concluyeron que para resolver esta duda sería necesario realizar investigaciones adicionales en el campo, complementadas con estudios experimentales en el ambiente controlado de un invernadero.

Durante 1998 y 1999 se realizaron varias expediciones al estado de Oaxaca en busca de poblaciones silvestres de las plantas conocidas con el nombre de $P$. 'alfredae', con la finalidad de recolectar especímenes para estudiarlos y resolver las dudas planteadas anteriormente; al mismo tiempo, se hicieron observaciones de las plantas en cultivo en el Jardín Botánico de Liberec en la República Checa. Esto nos permitió comprobar que se trata de dos especies independientes, que se diferencian en la morfología floral, en el comportamiento fenológico y en sus preferencias ecológicas. A continuación se describe formalmente la especie nueva y se discuten sus diferencias con Pinguicula heterophylla.

Pinguicula medusina Zamudio et Studnicka sp. nov. Fig. 1.

Herba perennis. Folia radicalia rosulata integerrima biformia; "rosula hiemis" hypogaea 9-23 mm longa, 9-20 mm lata bulbiformis ex pluribus foliis numerosis (70-90) composita, foliis exterioribus squamiformibus lanceolatis vel anguste lanceolatis acutis vel acuminatis glabriusculis marginibus longe ciliatis $8-23 \mathrm{~mm}$ longis $1.5-3.5 \mathrm{~mm}$ latis, intimis similibus sed non ciliatis; "rosula aestatis" 6-10(12) foliis initio erecta basi longe ciliata superne glandulis sessilibus dense et glandulis stipitatis modice dense vestita, foliis exterioribus (transitionis) 2-3(4) obovato-oblongis vel oblongo-lanceolatis obtusis vel acutiusculis $20-40 \mathrm{~mm}$ longis $3-7.5 \mathrm{~mm}$ latis, intimis longe lineari-lanceolatis gradatim in acumen tenue circinatum abeuntibus $70-190 \mathrm{~mm}$ longis $1.5-3.5 \mathrm{~mm}$ latis margine valde revolutis, gemmas in apicem formantibus. Pedunculi 1-3 erecti glandulis stipitatis obsiti 40$140 \mathrm{~mm}$ alti uniflori. Flores 16-23 mm longi (calcare incluso). Calyx bilabiatus extus glandulis stipitatis obsitus; labium superum usque ad basim trilobum, lobis triangulatis; labium inferum bilobum lobis usque ad 2/3 longitudinis divisis, lobis triangulatis. Corolla subisoloba alba vel alba violaceo marginata extus glandulis stipitatis disperse obsita lobis oblongis vel anguste-obovatis truncatis vel rotundatis $5-9 \mathrm{~mm}$ longis $2.5-5 \mathrm{~mm}$ latis. Tubus cylindricus sine palato albus vel violaceus ventraliter maculis flavovirentibus 6-9 mm longus intus pilosus basin tubi versus pilis brevioribus irregulariter capitatis. Calcar subcylindricum breve rotundatum 3-5 mm longum, cum tubo subporrectum. Stamina $\pm 2 \mathrm{~mm}$ longa; pollen (4)-5(6)-colporatum. Ovarium subglobosum glandulis stipitatis disperse obsitum. Stigma 
Zamudio y Studnicka: Nueva Especie Gipsícola de Pinguicula del Estado de Oaxaca, México

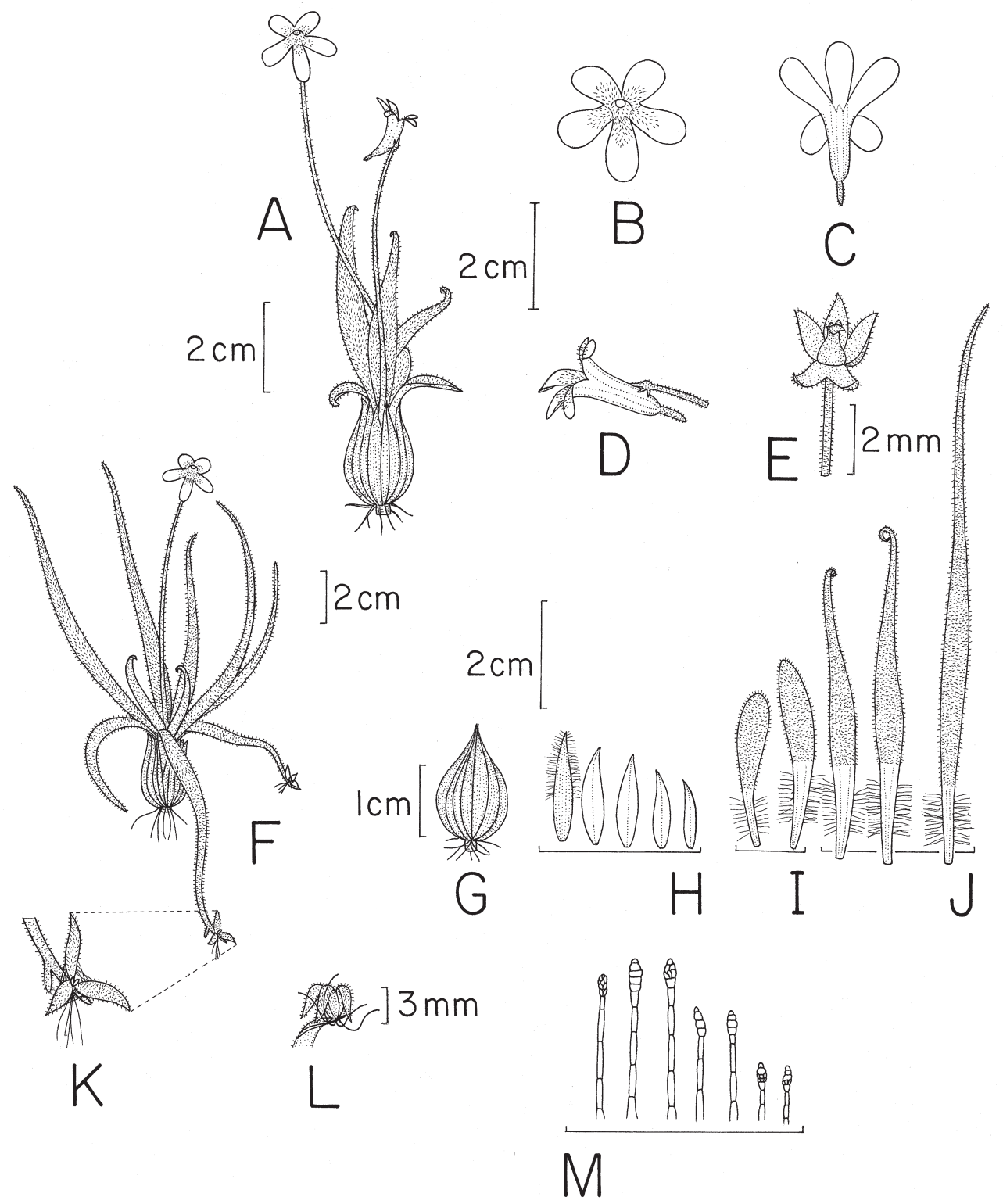

Fig. 1. Pinguicula medusina. A. Hábito de la planta en floración; B. Corola vista de frente; C. Vista posterior de la corola; D. Vista lateral de la corola; E. Cáliz; F. "Roseta de verano"; G. "Roseta de invierno"; H. Serie de "hojas de invierno"; I. Hojas transicionales; J. "Hojas de verano"; K. Yema en el ápice de la hoja; L. Yema desarrollada; M. Pelos del tubo de la corola. Dibujo de Rogelio Cárdenas. 
violaceum bilabiatum labio infero suborbiculato dentato alae angustae dentatae formans. Capsula subglobosa $\pm 3 \mathrm{~mm}$ diametro. Semina fusiformia $\pm 1 \mathrm{~mm}$ longa $\pm 0.25 \mathrm{~mm}$ lata, alveolata.

Planta herbácea perenne. Hojas basales, simples, dimórficas, agrupadas en dos rosetas subsecuentes; "roseta de invierno" hipogea, compacta en forma de bulbo, de 9 a $23 \mathrm{~mm}$ de largo, por 9 a $20 \mathrm{~mm}$ de ancho, formada por 70 a 90 hojas crasas, lanceoladas a angostamente lanceoladas, ápice agudo a acuminado, glabras, de 8 a $23 \mathrm{~mm}$ de largo, por 1.5 a $3.5 \mathrm{~mm}$ de ancho, las externas ciliadas con tricomas multicelulares de $\pm 5 \mathrm{~mm}$ de largo, escariosas en seco, las hojas interiores desprovistas de cilios; "roseta de verano" con 6 a 10(12) hojas membranáceas, más o menos erectas al principio y después adpresas al suelo, lanceolado-lineares, de 70 a $190 \mathrm{~mm}$ de largo, por 1.5 a $3.5 \mathrm{~mm}$ de ancho en la base, atenuadas hacia el ápice, con vernación circinada, margen fuertemente revoluto, densamente glandular-pubescentes en el haz con glándulas cortamente estipitadas (menos de $0.5 \mathrm{~mm}$ ), ciliadas en la base con tricomas multicelulares mayores de $5 \mathrm{~mm}$ de largo, las "hojas de verano" maduras forman yemas en el ápice, que dan origen a nuevas plantas por multiplicación vegetativa, hojas transicionales entre las de "invierno" y las de "verano" 2-3(4), obovado-oblongas a oblongo-lanceoladas, de 20 a $40 \mathrm{~mm}$ de largo, por 3 a $7.5 \mathrm{~mm}$ de ancho, obtusas o agudas. Pedúnculos 1 a 3, rollizos, de 40 a $140 \mathrm{~mm}$ de largo, densamente glandular-pubescentes. Flores de 16 a $23 \mathrm{~mm}$ de largo (incluyendo el espolón); cáliz bilabiado, el labio superior dividido casi hasta la base en tres lóbulos triangulares, de 1 a $2 \mathrm{~mm}$ de largo, por 0.5 a $1 \mathrm{~mm}$ de ancho, labio inferior bilobado hasta $2 / 3$ de su longitud, lóbulos triangulares, de 1 a $2 \mathrm{~mm}$ de largo, por 0.5 a $1 \mathrm{~mm}$ de ancho. Corola subisoloba, blanca o blanca con una franja de color lila en el ápice de los lóbulos, cubierta en la parte externa con glándulas estipitadas esparcidas; labio superior bilobado, los lóbulos en posición horizontal, sin retraerse fuertemente, oblongos a angostamente obovados, truncados a redondeados en el ápice, de 5 a $8 \mathrm{~mm}$ de largo, por 2.5 a $5 \mathrm{~mm}$ de ancho; labio inferior trilobado, un poco más grande que el superior, lóbulos oblongos a angostamente obovados, truncados o redondeados en el ápice, de 5 a $9 \mathrm{~mm}$ de largo, por 2.5 a $5 \mathrm{~mm}$ de ancho; tubo cilíndrico, desprovisto de paladar, de 6 a $9 \mathrm{~mm}$ de largo, blanco o ligeramente teñido de morado, con una mancha verde-amarillenta en la parte ventral, piloso en su interior con pelos multicelulares, irregularmente capitados, los pelos se observan más cortos hacia la base del tubo; espolón corto, subcilíndrico, de 3 a $5 \mathrm{~mm}$ de largo, redondeado en el ápice, subrecto en relación con el tubo, de color blanco. Estambres dos, de $\pm 2 \mathrm{~mm}$ de largo, polen (4)-5-(6)-colporado. Ovario subgloboso, cubierto con glándulas estipitadas esparcidas, estigma bilabiado, labio inferior suborbicular. Cápsula subglobosa, de $\pm 3 \mathrm{~mm}$ de diámetro. Semillas numerosas, fusiformes, de $\pm 1 \mathrm{~mm}$ de largo y $\pm 0.25 \mathrm{~mm}$ de ancho, alveoladas.

TIPO: México, Oaxaca, municipio de Juxtlahuaca; Laguna Encantada, $\pm 3 \mathrm{~km}$ al $\mathrm{N}$ de Santiago Juxtlahuaca, 27.V.1999, S. Zamudio y G. Ocampo 11050 (IEB).

Otros ejemplares revisados: México, Oaxaca, municipio de Juxtlahuaca; Laguna Encantada, $\pm 3 \mathrm{~km}$ al N de Santiago Juxtlahuaca, 22.XI.1998, S. Zamudio y G. Ocampo 10977 (IEB, ejemplares estériles con "hojas de verano"). 
Fenología: La floración se presenta de finales de mayo a julio, el desarrollo de la roseta de verano se inicia a fines de mayo, con el crecimiento de 2 a 3(4) hojas transicionales, de forma obovado-oblonga a oblongo-lanceolada, más cortas que las "hojas de verano", que permanecen por poco tiempo. Las "hojas de verano" linear-lanceoladas se pueden observar desde julio hasta noviembre o diciembre, cuando se forma la "roseta de invierno"; ésta es compacta, con forma de bulbo y permanece enterrada, protegida por una capa de hojas secas de consistencia escariosa, hasta mayo del siguiente año.

Es notable en $P$. medusina la formación de yemas en el ápice de las "hojas de verano", lo que le permite propagarse por multiplicación vegetativa. Las hojas de verano de la nueva especie crecen erectas al principio, pero se retraen conforme se desarrollan y finalmente quedan pegadas al suelo en la madurez. Las hojas se dividen dicotómicamente en el ápice, la división es asimétrica ya que sólo una de las partes se desarrolla y la otra queda reducida; las plantas nuevas se forman únicamente en la rama mayor y pronto producen numerosas raices que les permiten fijarse al suelo. Las plántulas originadas de esta forma cesan su actividad durante el invierno, adoptando un período de latencia que les capacita para soportar la temporada seca con éxito, para continuar su crecimiento cuando la humedad del suelo se restablece en la primavera del próximo año.

La ocurrencia de este mecanismo de propagación vegetativa es un caso único en el género, ya que hasta el momento no se conocía en ninguna otra especie. En la literatura sólo se ha registrado la formación de bulbilos axilares en Pinguicula alpina L., P. grandiflora Lam., $P$. longifolia Ram. ex DC. y $P$. vulgaris L.; mientras que en otras como $P$. calyptrata H.B.K., $P$. longifolia Ram. ex DC., $P$. orchidioides DC. y $P$. vallisneriifolia Webb. se menciona la formación de estolones con bulbilos (Casper, 1966; Luhrs, 1995; Zamudio, 1998).

Hábitat: Crece sobre colinas yesosas, en laderas inclinadas, sombreadas y húmedas, con vegetación de matorral rosetófilo y bosque tropical caducifolio, con Bursera schlechtendalii, B. mirandae, Vallesia sp., Dasylirion sp., Agave sp., entre 1600 y 1700 m de altitud.

Distribución: Hasta ahora sólo se conoce de la localidad tipo en los alrededores de la Laguna Encantada, $\pm 3 \mathrm{~km}$ al $\mathrm{N}$ de Santiago Juxtlahuaca, municipio de Juxtlahuaca, Oaxaca.

Discusión: En 1992 Van Marm y Lampard hicieron notar que tanto Pinguicula heterophylla como $P$. "alfredae" (ahora descrita como $P$. medusina) tienen hojas lineares parecidas a las de $P$. gypsicola Brandegee; sin embargo por sus flores con corola subisoloba, tubo cilíndrico y espolón más corto que el tubo, se ubican en la sección Heterophyllum (subgénero Isoloba) propuesta por Casper (1966), muy alejadas de la sección Orcheosanthus (subgénero Pinguicula) a la que pertenece $P$. gypsicola, lo que sugiere que la similitud en la forma de las "hojas de verano" es resultado de convergencia evolutiva.

Pinguicula "alfredae", ahora Pinguicula medusina muestra una fuerte semejanza con $P$. heterophylla, con la que comparte la mayoría de sus caracteres; por lo anterior se había puesto en duda si debería ser reconocida como una variedad de $P$. heterophylla o como una especie independiente (Van Marm y Lampard, 1992). 
Si bien a primera vista parece difícil separarlas, existen claras diferencias en la flor y en el hábitat que ocupan. En general, la flor de $P$. medusina es un poco más chica y delgada que la de $P$. heterophylla. La posición de los lóbulos de la corola difiere notoriamente pues, en $P$. heterophylla los del labio superior se retraen fuertemente, mientras que en $P$. medusina no se han observado retraídos. En $P$. heterophylla los lóbulos del labio inferior de la corola son obovado-oblongos, redondeados en el ápice y con frecuencia se sobreponen en los lados, en $P$. medusina en cambio son oblongos a angostamente obovados, truncados a redondeados en el ápice y no se sobreponen en los lados (Fig. 2).
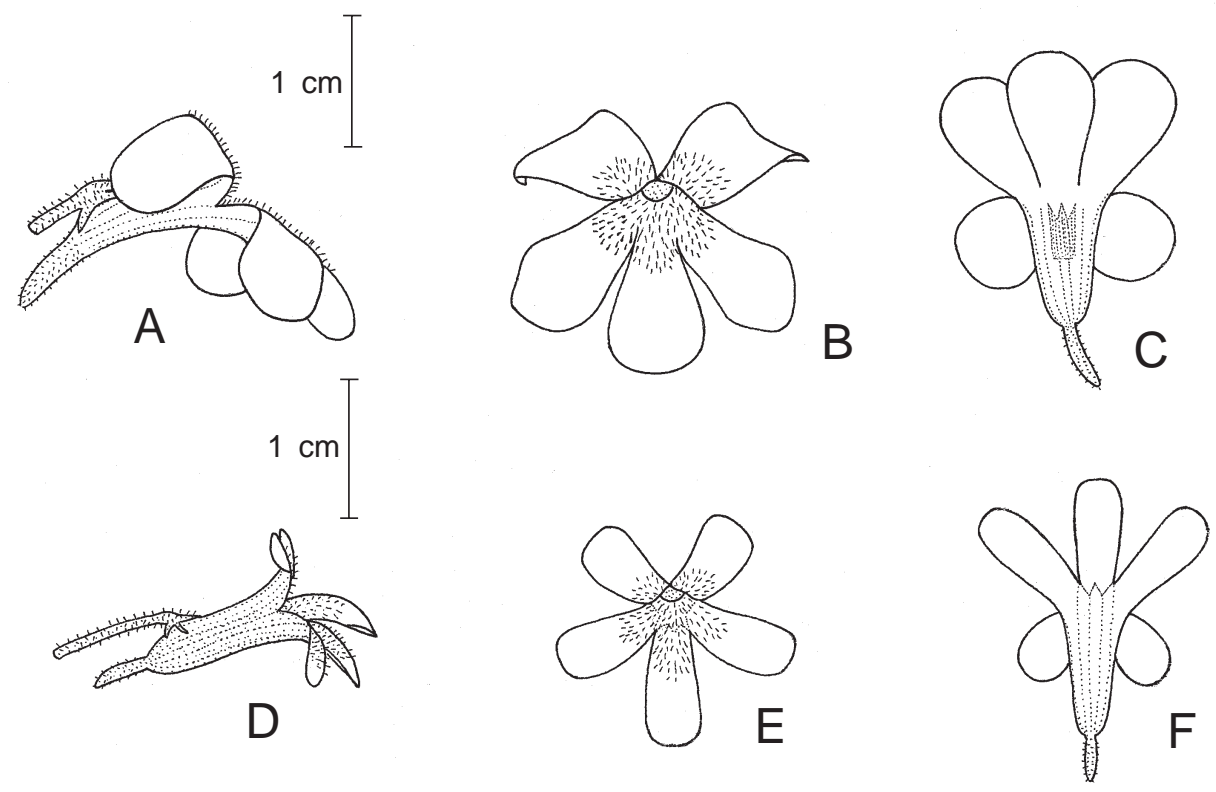

Fig. 2. Comparación de la flor de Pinguicula heterophylla A-C. y Pinguicula medusina D-F. Dibujo de Rogelio Cárdenas.

El patrón de coloración de la flor también es distinto: en $P$. heterophylla la corola es de color lila o blanquecina, con una mancha amarillo-verdosa en la garganta y el espolón verde claro; en cambio, en $P$. medusina la corola es blanca, o blanca con el margen de los lóbulos de color lila, el tubo es blanco o teñido de morado con una mancha verdeamarillenta en la parte ventral y el espolón blanco.

Cuando se cultivan individuos de las dos especies bajo las mismas condiciones, se observan diferencias en el comportamiento fenológico: $P$. heterophylla presenta flores de mayo a principios de junio, mientras ostenta hojas transicionales entre la "roseta de invierno" 
y la de "verano", las "hojas de verano" se hacen evidentes de finales de junio a finales de octubre, cuando se inicia la formación de la "roseta de invierno", que permanece hasta abril del próximo año; por su parte, en $P$. medusina el período de floración se extiende de principios de junio a principios de julio, como en el caso anterior, con la presencia de hojas transicionales, las que se mantienen hasta mediados de agosto, las "hojas de verano" se desarollan de agosto a diciembre y en enero dan paso a la formación de la "roseta de invierno", la que se conserva hasta junio (Cuadro 1).

Cuadro 1. Diferencias en el comportamiento fenológico entre Pinguicula medusina y $P$. heterophylla mantenidas en cultivo.

\begin{tabular}{|l|c|c|c|c|c|c|c|c|c|c|c|c|}
\hline & $\mathrm{E}$ & $\mathrm{F}$ & $\mathrm{M}$ & $\mathrm{A}$ & $\mathrm{M}$ & $\mathrm{J}$ & $\mathrm{J}$ & $\mathrm{A}$ & $\mathrm{S}$ & $\mathrm{O}$ & $\mathrm{N}$ & $\mathrm{D}$ \\
\hline P. medusina & & 000000 & & & \\
\hline P. heterophylla & & 0000 & \\
\hline
\end{tabular}

Roseta de invierno $\square$ Hojas transicionales $\square \quad$ Roseta de verano $\square$ Floración 000

En condiciones naturales el desplazamiento en las diferentes etapas fenológicas durante el ciclo anual entre las dos especies es menos marcado que en cultivo, en $P$. medusina la producción de flores dura aproximadamente un mes y se concentra de finales de mayo a principios de julio y la formación de la roseta de invierno se aprecia desde noviembre. En cambio, en $P$. heterophylla la etapa de floración se extiende por más de tres meses (de mayo a principios de agosto).

Algunas diferencias se han observado en las "rosetas de verano" de las plantas cultivadas: antes del desarrollo de las hojas linear-lanceoladas se aprecia la existencia de 2 a 3(4) hojas transicionales en Pinguicula medusina, mientras que en $P$. heterophylla se pueden encontrar hasta siete hojas de este tipo. Cuando la "roseta de verano" alcanza la madurez, puede estar formada por 6 a 12 hojas en $P$. medusina, y por 15 a 20 en $P$. heterophylla. Otra diferencia significativa es que en Pinguicula medusina se producen yemas en el ápice de las "hojas de verano", que dan origen a nuevas plantas por reproducción vegetativa, mientras que en $P$. heterophylla este fenómeno no se presenta.

También se observan claras diferencias en el hábitat que ocupan: $P$. heterophylla se ha colectado en bosque de encino, bosque de pino o bosque mixto de pino-encino, crece sobre suelos de color café-rojizo, originado a partir de rocas ígneas andesíticas o metamórficas graníticas, en un amplio intervalo altitudinal que va de 1500 a 3000 m s.n.m., en los estados de Michoacán, Guerrero y Oaxaca. Por su parte $P$. medusina sólo se conoce de la localidad tipo, en sitios con vegetación de matorral rosetófilo o bosque tropical caducifolio, sobre suelos someros derivados de rocas yesosas, entre 1600 y 1700 m s.n.m.

Aunque probablemente $P$. medusina y $P$. heterophylla comparten un antecesor común, $P$. medusina se ha adaptado a crecer sobre suelos yesosos en ambientes más secos, lo que le ha permitido diferenciarse y seguir un rumbo evolutivo propio. 
Etimología: El nombre de la especie hace referencia a la forma de la planta con una base bulbosa y largas hojas lineares, que recuerda la cabeza de Medusa, en la mitología griega una de las hijas de Forcis, dios del mar, que según la leyenda, tenía serpientes por cabellos.

\section{AGRADECIMIENTOS}

Los autores agradecen cumplidamente al Dr. Jerzy Rzedowski y a Rosa María Murillo la revisión crítica del manuscrito y sus atinadas observaciones, a Gilberto Ocampo su ayuda en la colecta de ejemplares botánicos.

\section{LITERATURA CITADA}

Casper, J. 1966. Monographie der Gattung Pinguicula L. Biblioth. Bot. 31(127-128): 1-209.

Luhrs, H. 1995. New additions to the genus Pinguicula (Lentibulariaceae) of Mexico. Phytologia 79(2): 114-122.

Van Marm, J. y S.E. Lampard. 1992. Some notes on Mexican pinguiculas. The International Pinguicula Study Group Newsletter 2: 12-16.

Zamudio, S. 1998. Situación taxonómica de Pinguicula orchidioides DC. (Lentibulariaceae). Acta Bot. Mex. 42: 7-13. 